\title{
Paediatric out-of-theatre procedural sedation at a tertiary children's hospital: A prospective observational study
}

\author{
C Lapere, DipPEC, DA (SA), PDD; R Gray, FCA (SA); G Wilson, FCA (SA) \\ Department of Anaesthesia and Peri-operative Medicine, University of Cape Town, South Africa \\ Corresponding author: C Lapere (cherese.lapere@gmail.com)
}

\begin{abstract}
Background. Red Cross War Memorial Children's Hospital (RCWMCH) is one of the largest paediatric hospitals in Africa. Despite an increasing number of surgical and diagnostic procedures being performed annually, a formal out-of-theatre sedation service does not exist. Procedural sedation and analgesia (PSA) is an important adjunct in behavioural management for invasive procedures in children.

Objective. A prospective, observational study was performed at RCWMCH, aimed primarily at defining the number of cases of PSA performed outside the operating theatre.

Methods. Data were collected over a period of 3 months from all procedural out-of-theatre sedations performed, including ward patients and outpatient departments. All children $<13$ years of age were included.

Results. A total of 639 sedations were performed. Of these sedations, 288 (45.1\%) paper responses were captured and analysed. The reported incidence of airway obstruction was $4.9 \%(n=14 / 288)$, desaturation $4.2 \%(n=12 / 288)$, laryngospasm $0.3 \%(n=1 / 288)$ and nausea and vomiting $2.4 \%(n=7 / 288)$. Three cases required conversion to general anaesthesia, and four cases were abandoned as a result of inadequate sedation. In $16.3 \%(n=47 / 288)$ of cases, the clinician was an operator sedationist (the same person performing the sedation and the procedure). In $90.6 \%$ of cases, the intravenous route was utilised, with dexmedetomidine, ketamine and propofol being the three most commonly used agents.

Conclusion. A total of 639 PSA events were recorded in 3 months. The 288 events analysed were safely performed with minimal serious reported events. These results compare favourably with international studies and provide quantitative evidence as a prelude to setting up a dedicated sedation service at RCWMCH.
\end{abstract}

S Afr J Child Health 2020;15(1):33-37. https://doi.org/10.7196/SAJCH.2020.v15i1.1775

Procedural sedation and analgesia (PSA) describes the use of agents, such as sedatives and analgesics, to alleviate anxiety, pain and fear during diagnostic and therapeutic procedures. ${ }^{[1]}$ The South African Society for Anaesthesiologists (SASA) defines the goals of procedural sedation as reducing the patient's fear, anxiety and distress while minimising physical discomfort, pain and psychological trauma, maintaining control of physiological parameters and ensuring patient safety. ${ }^{[2]}$ Inadequate pain management in the paediatric population has the potential for lasting negative consequences and therefore adequate pain management is an essential part of PSA. ${ }^{[3,4]}$ The use of PSA has been validated and extensively researched as an adjunct to facilitate procedures in the paediatric population. ${ }^{[4-6]}$

Procedural sedation is a continuum. It ranges from mild sedation, through to moderate and deep sedation, and finally culminates in the induction of general anaesthesia. ${ }^{[2]}$ Sedation should be considered as a dose-dependent change in the level of consciousness that should be titrated according to the patient's response by administration of the minimum amount necessary to avoid unintended deeper levels of sedation. ${ }^{[7]}$ Diagnostic and therapeutic procedures are often facilitated with the performance of moderate to deep sedation, with the patient or the procedure dictating the intended level of sedation required. ${ }^{[4]}$ Unfortunately there is currently no single risk-free sedative that fulfils all the criteria as the perfect agent for PSA. ${ }^{[8]}$

PSA has been performed in various locations throughout our paediatric tertiary referral hospital by anaesthetists and nonanaesthetists. This study sought to understand the nature and extent of PSA provision in the hospital as a precursor to setting up a formalised out-of-theatre sedation service.

\section{Methods}

A prospective observational study of all consecutive patients $<13$ years of age requiring PSA for diagnostic or therapeutic indications outside the operating theatre was performed at Red Cross War Memorial Children's Hospital (RCWMCH), a paediatric, tertiary-level referral centre in Cape Town, South Africa (SA). The primary objective was to determine the number of sedations performed. PSA performed in the intensive care unit and the trauma unit were excluded from this study as they would not fall within the remit of an out-of-theatre sedation service were it to be established.

During the time of the study, the number of cases was measured by a twice-weekly audit of the scheduled drug books in each ward and outpatient area. Medications known to be used for PSA in each area (dexmedetomidine, propofol, ketamine, midazolam, etomidate, fentanyl or chloral hydrate) were audited and patient hospital numbers correlated with records of procedural sedations kept by the unit manager in each area/ward. The hospital identification numbers were not recorded to preserve anonymity.

Attending clinicians completed a data capture sheet specific to the study after each sedation event and this was used to generate the secondary outcomes of the study. Clinician participation in the study was voluntary and no clinician or patient identifiers were collected. 
Information collected included the child's age group, comorbidities, hospital location, and level of expertise of staff performing the sedation, as well as occurrence of adverse events. The choice of drug and route of administration, as well as the type of monitoring, were also captured. Recorded adverse events included those related to the airway, the quality of the sedation and other known drug-specific adverse events of the sedatives (such as nausea and vomiting) administered.

Privacy and confidentiality of collected data were ensured at all times. On-paper data were electronically captured on RedCap, a password-protected and encrypted platform specifically used for management of electronic data and research. Descriptive statistical analysis was done using the SPSS version 25 (IBM Corp., USA) statistical package.

This prospective observational cohort was approved by the Human Research Ethics Committee of the Faculty of Health Sciences at the University of Cape Town (ref. no. 404/2018). Institutional permission was also obtained to conduct research at the facility (ref. no. RXH-RCC-174).

\section{Results}

During the 3-month enrolment period (18 February to 19 May 2019), out-of-theatre sedations were provided for 639 procedures at RCWMCH. PSA was administered in a variety of different locations across the facility during this study period. Overall, $36.3 \%$ of total sedations $(n=232)$ took place in the wards, and $30.7 \%(n=196)$ in the radiology suite on either outpatients or inpatients. The acute service (admission and acute care area) performed 180 sedations (28.2\%) and the outpatient department performed 31 (4.9\%) sedations.

Two hundred and eighty-eight (45.1\%) of the sedations performed were captured on data sheets by the attending staff. Data collection was complete and could be analysed for all 288 patients. Secondary outcomes for this study are reported for these 288 patients. Overall, $61.1 \%$ of captured sedations $(n=176)$ took place in the radiology suite on either outpatients or inpatients. The acute service reported on 26 (9.0\%) of the sedations, while the outpatient/ clinic department reported $21(7.3 \%)$ and the combined number of sedations reported in the wards totalled $65(22.6 \%)$. Table 1 outlines the patient demographics and comorbidities of these cases.

Sedation events captured by clinicians showed that these were most frequently performed by a practitioner who, at the time, only managed the sedation, termed 'sedation provider' (Table 2).

Table 3 summarises the route of administration and the most commonly used drugs during PSA. Intravenous administration of sedatives was the most utilised route, followed by oral sedatives.

Fig. 1 summarises the monitors applied. The majority of sedations (95.1\%) were performed with, at minimum, pulse oximetry, while $4.5 \%$ of cases were performed with no monitoring.

Sixty adverse events were reported. The two most frequently reported adverse events were desaturation, which occurred in $4.2 \%$ of recorded sedations, and airway obstruction requiring manipulation of the airway (4.9\% (Table 4). Other adverse events include nausea and vomiting, or the necessity to either abort the sedation or convert to general anaesthesia.

\section{Discussion}

With only six full-time consultant anaesthetists and six registrars, the complex provides anaesthesia to over 8500 children per annum. ${ }^{[9]}$ An additional out-of-theatre service inevitably places an extra burden on an already overstretched system.

A growing number of procedures are performed outside the operating room and therefore the demand for anaesthesia outside the traditional operating theatre has increased significantly. ${ }^{[10]}$ Although serious adverse events are rare, SASA emphasises the responsibility of all healthcare professionals involved in sedation to be accountable for safe practice. The patient is entitled to the same degree of vigilance and attention to the principles of safe practice, whether the procedure is undertaken in the office, surgery, a remote facility or operating theatre. ${ }^{[2]}$

\section{Rates of adverse events}

Owing to the low incidence of major adverse outcomes, such as permanent neurological damage or death, studies that target PSA outcomes need to choose different criteria to measure safe and successful procedural outcomes. ${ }^{[8,11-13]}$ Unfortunately, reports on sedation outcomes are complicated by a lack of standardisation of adverse events with inconsistent definitions resulting in over- or under-reporting thereof. ${ }^{[11]} \mathrm{An}$ example is hypoxia which may be defined by oxygen saturations $<93 \%, 90 \%, 85 \%$ for 30 or 60 seconds. ${ }^{[11]}$ In a collaborative effort by various societies the standardisation of the definitions of adverse events has been prioritised by the International Committee for the Advancement of Procedural Sedation (ICAPS). Their definition of an adverse event during PSA is an undesirable response to medical interventions or medicine causing patient injury or discomfort. ${ }^{[11,13]}$

A systematic review and meta-analysis of adverse events in the emergency department when performing PSA considered 13883 sedations and found serious adverse events to be very rare. ${ }^{[8]}$ The total rate of intubation was $0.04 \%$ and reported laryngospasm $0.29 \%$. The rate of intubation reported in this study is significantly higher $(0.7 \%)$, but also included patients who were converted to general anaesthesia and required secured airway management.

The referenced incidence of complications arising in the paediatric population during PSA is $0.8-9 \%$ for hypoxaemia, depending on the definition used. ${ }^{[14]}$ In this study hypoxaemia was defined as an oxygen, saturation level $<90 \%$ for $>60$ seconds. The recorded rate was $4.1 \%$, which falls within the internationally reported range, but is markedly higher than the estimated rate per $1000(1.4 \%)$ cases in the aforementioned review. ${ }^{[8]}$ This may be explained by the severity of comorbidities within the patient population of a paediatric tertiary referral hospital, but could also be explained by the fact that a large proportion of analysed cases were performed in the radiology unit where the patient was expected to be supine and immobile with little to no stimulation for extended periods.

One of the current benchmarks of adverse airway event reporting is the 4th National Audit Project of the Royal College of Anaesthetists and Difficult Airway

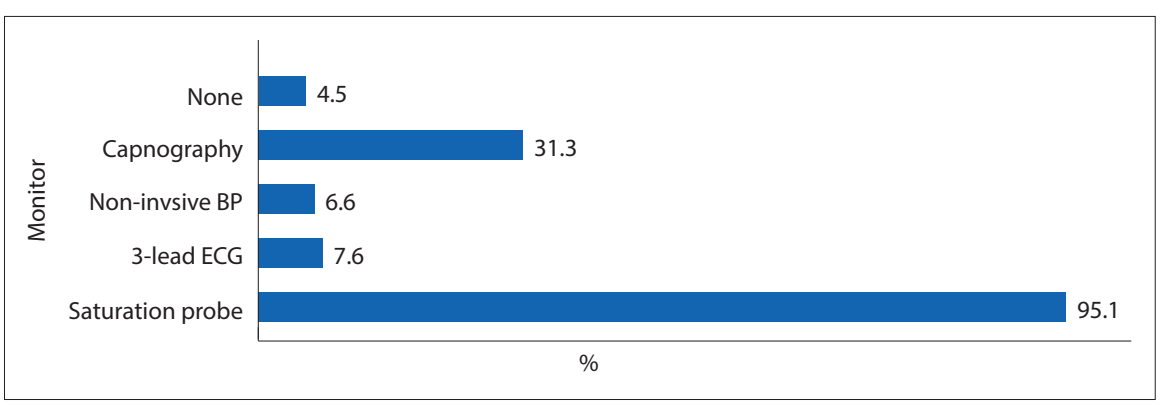

Fig. 1. Monitors applied. $(B P=$ blood pressure; $E C G=$ electrocardiograph. $)$ 


\begin{tabular}{|c|c|}
\hline & $N=288, n(\%)$ \\
\hline \multicolumn{2}{|l|}{ Age } \\
\hline$<1$ month & $2(0.7)$ \\
\hline$\leq 1$ year & $51(17.7)$ \\
\hline$\leq 5$ years & $136(47.2)$ \\
\hline$>5$ years & $99(34.4)$ \\
\hline \multicolumn{2}{|c|}{ Comorbidities of patients } \\
\hline Cardiac & $17(5.9)$ \\
\hline Respiratory & $23(8.0)$ \\
\hline Renal & $22(7.6)$ \\
\hline Neurological & $126(43.8)$ \\
\hline Airway & $8(2.8)$ \\
\hline Other & $49(17.0)$ \\
\hline None & $82(28.5)$ \\
\hline
\end{tabular}

Table 2. Performing clinician's level of expertise and involvement

\begin{tabular}{ll}
\hline involvement & $n(\%)$ \\
\hline Role of sedation provider & \\
Sedation provider only & $241(83.7)$ \\
Operator seditionist & $47(16.3)$ \\
Level of expertise of sedation provider & \\
Consultant & $164(56.9)$ \\
Registrar & $48(16.7)$ \\
MO & $36(12.5)$ \\
Community service MO & $7(2.4)$ \\
Intern & $23(8.0)$ \\
Nurse & $10(3.5)$ \\
MO = medical officer. &
\end{tabular}

Table 3. Description of the sedative and route

\begin{tabular}{ll}
\hline & $n(\%)$ \\
\hline Sedatives & \\
Dexmedetomidine & $144(50.0)$ \\
Ketamine & $118(41.0)$ \\
Propofol & $107(37.2)$ \\
Midazolam & $30(10.4)$ \\
Chloral hydrate & $21(7.3)$ \\
Sevoflurane & $13(4.5)$ \\
Opioids & $5(1.7)$ \\
Other & $4(1.4)$ \\
Droperidol & $1(0.3)$ \\
Route of sedative & \\
Intravenous & $261(90.6)$ \\
Oral & $26(9.0)$ \\
Inhalation & $24(8.3)$ \\
Local/topical & $8(2.8)$ \\
Nasal & $5(1.7)$ \\
Rectal & None \\
&
\end{tabular}

Society (NAP4). According to NAP4, human factors that most commonly contribute to potentially avoidable airway complications are inadequate training and education, as well as poor clinical judgement and appreciation of the unique paediatric airway. ${ }^{[15]}$
Table 4. Description of reported adverse events

\begin{tabular}{ll}
\hline \multicolumn{2}{l}{ Table 4. Description of reported adverse events } \\
\hline Airway-related events & $n(\%)$ \\
Desaturation (Sats $<90 \%$ for 60 s or longer) & $12(4.2)$ \\
Airway obstruction requiring any airway & $14(4.9)$ \\
manipulation & \\
Use of airway adjuncts (oral, nasal) & $3(1.0)$ \\
Hypoventilation requiring additional oxygen & $3(1.0)$ \\
Patient required bag-valve-mask ventilation & $4(1.4)$ \\
Patient required an advanced airway (ETT, & $2(0.7)$ \\
LMA) & $1(0.3)$ \\
Laryngospasm & None \\
Bronchospasm & \\
Other & $5(1.7)$ \\
Dysrhythmia: presence of a bradycardia or & \\
tachycardia, or other & None \\
Hypotension requiring an intervention & $7(2.4)$ \\
Nausea and/or vomiting & $2(0.7)$ \\
Emergence delirium or aggression & None \\
Allergic reaction & $4(1.4)$ \\
Child not co-operative and sedation aborted & $3(1.0)$ \\
Sedation converted to general anaesthesia & \\
Sats = oxygen saturation; ETT = endotracheal tubation; & \\
LMA = laryngeal mask airway. &
\end{tabular}

Paediatric patients are distinctly different from adults in behaviour, development and physiology, with anatomical differences that make them more prone to adverse events during anaesthesia. Children often require a deeper level of sedation than adults in order for the procedure to be completed. Because of their higher metabolic demand, they are at greater risk of hypoxaemia and hypercarbia during periods of apnoea. ${ }^{[8]}$

In paediatric PSA, failed sedation may result from relative oversedation or poor titration leading to adverse respiratory events, or from insufficient sedation, resulting in an agitated, undersedated patient. ${ }^{[4,16]}$ The present study reported 7 cases $(2.4 \%)$ of failed sedation with 4 cases aborted and 3 converted to general anaesthesia. Factors known to contribute to failure of sedation include children with comorbidities classified as American Society of Anesthesiologists (ASA) 3 or 4 , use of single agents for sedation, mentally retarded children, and those with autism. ${ }^{[14]}$ In the present study $0.7 \%$ of children were reported to be restless or aggressive compared with a report of restlessness of $1.8 \%$ in children undergoing PSA in the emergency department. ${ }^{[8]}$

The combination of the safest and most effective sedative is yet to be established. ${ }^{[8]} \mathrm{A}$ large multicentre observational study investigating risk factors for adverse events during sedation found that the incidence varied significantly with the type of medication used. Ketamine alone was associated with the least amount of adverse events compared with multidrug sedations. ${ }^{[17]}$ Oxygenation and ventilation is the most critical function of airway management. Administration of sedatives, including benzodiazepines (Dexmedetomidine was not included in this study), propofol and opioids, can cause an increased risk of airway obstruction due to exacerbation of pharyngeal collapsibility. ${ }^{[18]}$ Dexmedetomidine has been reported to induce less airway collapsibility and respiratory depression. ${ }^{[19]}$ The pharyngeal musculature tone and the airway reflexes are also largely preserved with the use of ketamine. ${ }^{[20]}$ Our low rate of adverse events could be explained by the choice of sedative, with dexmedetomidine and ketamine being the most utilised drugs. In addition, the intravenous 
route (most frequently used in this study) also lends itself to careful titration of drugs, which in itself is hypothesised to contribute to fewer adverse events. ${ }^{[12]}$

The SASA Paediatric Sedation Guidelines for Procedural Sedation and Analgesia define advanced sedation as the use of a combination of sedative drugs, the administration of one or more sedative agents via the intravenous route or the use of inhalational sedation (excluding nitrous oxide at $50 \%$ or less). ${ }^{[21]}$ Advanced sedation requires a dedicated sedationist with appropriate clinical and life support training to work alongside the proceduralist.

\section{Level of expertise of practitioners performing PSA}

The majority of captured sedations were performed in the radiology unit by consultant anaesthetists. Syndromic children and those with significant neurological comorbidities (both factors recognised to predict risk of adverse events) comprise a substantial portion of the patients requiring MRI, therefore this is a service provided by the Anaesthesia Department.

SA has a critical shortage of anaesthesiologists while healthcare centres are experiencing a rise in the number of paediatric patients requiring sedation. The non-anaesthetist is essential in providing PSA for paediatric patients. ${ }^{[16]}$ A North American survey showed that structured sedation programmes were rare at many facilities; ${ }^{[22]}$ however, it is known that non-anaesthetists, including a wide variety of different specialists, are providing safe paediatric sedation, utilising various routes of administration and drugs. ${ }^{[4,10,23]}$ With careful patient selection and preselected drug regimens, a nurselead sedation service potentially allows physicians to tend to other clinical duties. Such a service could prove to be a very important adjunct in a resource-constrained institution.

The sedation practitioner needs to be able to manage the sedation, as well as medical emergencies that might occur during the sedation. ${ }^{[2,12]}$ Patients with underlying medical conditions and the very young are at greater risk of adverse events; an even greater degree of vigilance is required in this group of children. ${ }^{[24]}$

The responsibility lies with the sedation providers to rescue patients that unintentionally slip into deeper levels of sedation. Collaboration between departments and training in sedation techniques are needed, especially in underdeveloped or rural areas. Such collaboration can address a lack of knowledge about paediatric sedation techniques, train clinicians about critical incidents and paediatric airway management, and highlight the factors contributing to the high risk/low error tolerance of children. ${ }^{[10]}$

\section{Monitoring}

During procedural sedation, patients need to be monitored with vigilance to identify an unintentional increase in sedation depth. Clinical monitoring of vital signs is important. Capnography has a role in detecting apnoea or airway obstruction due to cessation of flow several minutes before a decrease in pulse oximetry is detected, and could enable earlier intervention. End-tidal carbon dioxide monitoring is not mandatory in SA during PSA; however, increasing evidence points to the safety it adds, especially in patients who are deeply sedated. Our results show that capnography was used during $31.3 \%$ of sedations. Thirteen cases $(4.5 \%)$ of PSA were performed without the use of any monitoring; this is in contravention of the SASA guidelines, which prescribe monitoring dependent on the planned depth of sedation or intended drug regimen. ${ }^{[21]}$

\section{Standard of care}

The standard of care for paediatric PSA is defined by the South African Paediatric Guidelines for Procedural Sedation and Analgesia. ${ }^{[21]}$ These peer-reviewed principles include drugs, dosages and combinations that can safely be employed by sedation practitioners. Monitoring, record-keeping, emergency equipment and minimum training are outlined. Paediatric-specific risk stratification methods will help practitioners establish the probability of adverse events and whether the case is suitable for out-of-theatre sedation in the hands of a non-anaesthetist.

\section{Study limitations}

The study has several limitations: our capture rate of data forms necessary for secondary outcomes was $45.0 \%$. We relied on external data collectors, clinicians performing the sedations, to complete the data forms while they were on duty in busy, often understaffed units, or nursing staff who performed the sedations while often being responsible for patient flow in the outpatient clinics. The extra burden of data collection during normal day-today duties may have contributed to our low capture rate. Owing to the relatively small number of patients, univariate analysis to identify independent risk factors for specific adverse events could not be performed. Had data collection covered the full number of performed sedations, the secondary outcomes might have been different.

Another potential limitation was not being able to calculate a sample size. We included all cases over a period of time and captured a cohort that would represent the current management. We did not include postsedation clinician or caregiver feedback, as well as patient (or caregiver where applicable) satisfaction in this study, but perhaps this could be incorporated in future projects.

\section{Conclusion}

The use of procedural sedation for diagnostic and therapeutic procedures is common in the facility. The reported incidence of adverse events in the $45 \%$ of cases in which this could be assessed is low and in line with international reports. Future projects will include the set-up and implementation of a dedicated sedation service with the standard of care detailed in the SA guidelines, and reporting on patient outcomes and satisfaction, as well as the possible positive impact that such a service might have on the hospital. Protocols, guidelines and training are the essential cornerstones of out-oftheatre procedural sedation.

\section{Declaration. None.}

Acknowledgements. Thank you to nursing and clinical staff who assisted with data collection and for guidance with the statistical analysis.

Author contributions. CL conceptualised the project, collected data, analysed results and wrote the first draft of the manuscript. RG and GW supervised the project and reviewed all drafts.

Funding. None.

Conflicts of interest. None.

1. Krauss B, Green SM. Procedural sedation and analgesia in children. Lancet 2006;367(9512):766-780. https://doi.org/10.1016/s0140-6736(06)68230-5

2. Roelofse J, Piercy J. Society of South African Society of Anaesthesiologists Sedation Guidelines 2015: Guidelines for the safe use of procedural sedation and analgesia for diagnostic and therapeutic procedures in adults: 2015. S Afr J Anaesth Analg 2015;21(2):1-38.

3. Chafe R, Harnum D, Porter R. Improving the treatment and assessment of moderate and severe pain in a pediatric emergency department. Pain Res Manag 2016. https://doi.org/10.1155/2016/4250109

4. Gozal D, Drenger B, Levin PD, Kadari A, Gozal Y. A pediatric sedation/ anesthesia program with dedicated care by anesthesiologists and nurses for procedures outside the operating room. J Pediatr 2004;145(1):47-52. https:// doi.org/10.1016/j.jpeds.2004.01.044 
5. Coté C, Wilson S, American Academy of Pediatrics, American Academy of Pediatric Dentistry. Guidelines for Monitoring and Management of Pediatric Patients Before, During, and After Sedation for Diagnostic and Therapeutic Procedures: Update 2016. Pediatrics 2016;138(1):180-185. https://doi. org/10.1542/peds.2016-1212

6. Monroe KK, Beach M, Reindel R, et al. Analysis of procedural sedation provided by pediatricians. Pediatr Int 2013;55(1):17-23. https://doi.org/10.1111/j.1442200x.2012.03743.x

7. Green SM, Krauss B. Clinical Practice Guideline for Emergency Department Ketamine Dissociative Sedation in Children. Ann Emerg Med 2004;44:460471. https://doi.org/10.1016/s0196064404006365

8. Bellolio MF, Puls HA, Anderson JL, et al. Incidence of adverse events in paediatric procedural sedation in the emergency department: A systematic review and meta-analysis. BMJ Open 2016;6(6). https://doi.org/10.1136/ bmjopen-2016-011384

9. Meyer HM, Thomas J, Wilson GS, de Kock M. Anesthesia-related and perioperative mortality: An audit of 8493 cases at a tertiary pediatric teaching hospital in South Africa. Pediatr Anaesth 2017;27(10):1021-1027. https://doi. org/10.1111/pan.13214

10. Mason KP, ed. Pediatric Sedation Outside of the Operating Room: A multispecialty International Collaboration (e-book). $2^{\text {nd }}$ ed. Springer, 2015;1-755.

11. Roback MG, Green SM, Andolfatto G, Leroy PL, Mason KP. Tracking and Reporting Outcomes Of Procedural Sedation (TROOPS): Standardised quality improvement and research tools from the International Committee for the Advancement of Procedural Sedation. Br J Anaesth 2018;120(1):164-172. https://doi.org/10.1016/j.bja.2017.08.004

12. Coté CJ, Karl HW, Notterman DA, Weinberg JA, McCloskey C. Adverse sedation events in pediatrics: Analysis of medications used for sedation. Pediatrics 2000;106(4):633-644. https://doi.org/10.1542/peds.106.4.633

13. Mason KP, Green SM, Piacevoli Q. Adverse event reporting tool to standardise the reporting and tracking of adverse events during procedural sedation: A consensus document from the World SIVA International Sedation Task Force. Br J Anaesth 2012;108(1):13-20. https://doi.org/10.1093/bja/aer407

14. Malviya S, Voepel-Lewis T, Eldevik OP, Rockwell DT, Wong JH, Tait AR. Sedation and general anaesthesia in children undergoing MRI and CT: Adverse events and outcomes. Br J Anaesth 2000;84(6):743-748. https://doi. org/10.1093/oxfordjournals.bja.a013586
15. Cook TM. Strategies for the prevention of airway complications: A narrative review. Anaesthesia 2018;73(1):93-111.

16. Campbell K, Torres L, Stayer S. Anesthesia and sedation outside the operating room. Anesthesiol Clin 2014;32(1):25-43. https://doi.org/10.1016/j. anclin.2013.10.010

17. Bhatt M, Johnson DW, Chan J, et al. Risk factors for adverse events in emergency department procedural sedation for children. JAMA Pediatr 2017;171(10):957-964. https://doi.org/10.1001/jamapediatrics.2017.2135

18. Connolly LA. Anesthetic management of obstructive sleep apnea patients. J Clin Anesth 1991;3(6):461-469. https://doi.org/10.1016/09528180(91)90094-4

19. Masin KP, O’Mahony E, Zurakowski D, Libenson MH. Effects of dexmedetomidine sedation on the EEG in children. Pediatr Anesth 2009;19(12):1175-1183. https://doi.org/10.1111/j.1460-9592.2009.03160.x

20. Drummond GB. Comparison of sedation with midazolam and ketamine: Effects on airway muscle activity. Br J Anaesth 1996;76(5):663-667. https:// doi.org/10.1093/bja/76.5.663

21. Roelofse JA, Gray RM. Paediatric sedation guidelines for procedural sedation and analgesia. S Afr J Anaesth Analg 2016;22(1 Suppl 5):1-33.

22. Lalwani K, Michel M. Pediatric sedation in North American children's hospitals: A survey of anesthesia providers. Pediatr Anesth 2005;15(3):209213. https://doi.org/10.1111/j.1460-9592.2005.01437.x

23. Newstead B, Bradburn S, Appelboam A, et al. Propofol for adult procedural sedation in a UK emergency department: Safety profile in 1008 cases. Br J Anaesth 2013;111(4):651-655. https://doi.org/10.1093/bja/aet168

24. Malviya S, Voepel-Lewis T, Tait AR. Adverse events and risk factors associated with the sedation of children by nonanesthesiologists. Anesth Analg 1997;85(6):1207-1213. https://doi.org/10.1097/00000539-19971200000005

Accepted 17 August 2020. 